\title{
INTELIGENCIA EMOCIONAL: AVANCES TEÓRICOS Y APLICADOS TRAS 30 AÑOS DE RECORRIDO CIENTÍFICO
}

\author{
Natalio Extremera, Sergio Mérida-López y Nicolás Sánchez-Álvarez \\ Universidad de Málaga, España
}

\section{EDITORIAL}

\section{Introducción}

Pocos constructos psicológicos en las últimas décadas han atraído la atención de psicólogos, educadores, formadores, y técnicos de recursos humanos como lo ha hecho el concepto de inteligencia emocional. Tras 30 años desde la aparición del concepto científico, presentamos este monográfico en la revista Know and Share Psychology en reconocimiento a sus padres conceptuales, Peter Salovey y John Mayer, por su contribución al estudio conjunto del campo de la inteligencia y las emociones. Esta recopilación de trabajos de España, Portugal, Colombia, México y EEUU, escritos por prestigiosos científicos e investigadores nacionales e internacionales de los cuales estamos orgullosos de poder decir que son buenos amigos, es un reconocimiento a la aportación de una nueva idea de inteligencia, que supuso un fructífero campo de investigación desde sus inicios y que ha llevado a su aplicación en múltiples áreas de la esfera humana. Anteriormente a este homenaje, otros monográficos han sido realizados en revistas internacionales como tributo a la inteligencia emocional a lo largo de estas décadas. Todos ellos han focalizado su interés en las contribuciones que el concepto y las habilidades emocionales que la componen han supuesto para su aplicación a diferentes campos de la psicología, influyendo directa e indirectamente en el bienestar, el rendimiento y la calidad de vida de las personas. Sin embargo, hasta nuestro conocimiento, ningún homenaje se ha realizado en su trigésimo aniversario en lengua española. Tal como se podrá apreciar en los trabajos que se presentan, la inteligencia emocional ha generado mucho interés en los países de lengua castellana, convirtiéndose en un área de investigación psicológica desarrollada y madura.

Aunque el término se utilizó anteriormente (Leuner, 1966), el origen científico surgió en 1990, de la mano de dos profesores americanos, Peter Salovey y John Mayer, quienes publicaron el primer artículo sobre inteligencia emocional en la revista Imagination, Cognition and Personality. Para introducir el término, los autores propusieron un conjunto de habilidades emocionales interrelacionadas apelando a que la inteligencia emocional era parte de una larga tradición en el campo de la inteligencia en la que algunos investigadores habían explorado ciertas subáreas tales como la esfera social (Thorndike, 1920) o intrapersonal (Gardner, 1983). Si bien inicialmente este primer artículo tuvo escasa repercusión científica, sin duda, Daniel Goleman (1995) con su libro y su justa mención de que el término lo tomaba prestado de estos dos profesores, fue un detonante a nivel mundial que sirvió para amplificar los efectos mediáticos y científicos de este concepto. A partir de ahí, el crecimiento de la investigación ha sido creciente y sustancial. Por ejemplo, desde la base de datos Thomson Reuters' Web of Science se ha observado un incremento paulatino de artículos científicos relacionados y, a partir de 2015, se ha superado la barrera de las 1000 publicaciones científicas anuales. Desde entonces, las publicaciones siguen creciendo en número cada año con nuevos y apasionantes debates concep-

Correspondencia.

\author{
Natalio Extremera \\ nextremera@uma.es
}

Departamento de Psicología Social, Trabajo Social, Antropología Social y Estudios de

Asia Oriental.

Facultad de Psicología, Universidad de Málaga 
tuales y aplicaciones prácticas. No obstante, la inteligencia emocional también ha convivido con la aparición de numerosas publicaciones no científicas que empañaron la cientificidad del constructo. De hecho, desde sus comienzos, el constructo recibió numerosas críticas que ha llevado a los científicos a aclarar aspectos conceptuales, de medición y de su importancia sobrevalorada en ciertas áreas vitales. Aunque aún sigue habiendo detractores y críticas más que razonables, son muchas las investigaciones que ya han demostrado la utilidad del constructo, la complementariedad entre diferentes enfoques teóricos, su interrelación con otros constructos y la posibilidad de que las habilidades que la componen sean entrenadas mediante propuestas sistemáticas y rigurosas de aprendizaje en diversas esferas de la vida humana.

Tras más de treinta años, Peter Salovey en la Universidad de Yale, John Mayer en la Universidad de New Hampshire, y sus respectivos equipos de investigación, han tratado de demostrar, con el rigor y la sistematicidad propia de la ciencia, la utilidad e importancia de las habilidades básicas integradas dentro de su modelo de procesamiento de la información emocional (Mayer et al., 2016). Es cierto que este no ha sido el único enfoque teórico de inteligencia emocional y debemos reconocer el valor de otros teóricos que han aportado sus acercamientos y su valioso soporte empírico, tales como el profesor Reuven Bar-On de la Universidad de Texas (EEUU), los profesores Adrian Furnham y Konstantinos Petrides de la Universidad de Londres (Inglaterra) o la profesora Moïra Mikolajczak de la Universidad Católica de Lovaina (Bélgica), entre otros. Gracias a estos múltiples acercamientos, se ha constatado la necesidad de un desarrollo teórico más integrador, que a través de datos y evidencias científicas permitan establecer modelos estructurales multi-nivel en el que los diferentes atributos de la inteligencia emocional, (auto-percepciones, conocimientos, rasgos y habilidades), se interrelacionan con otras destrezas y predisposiciones esenciales del ser humano (p.ej., rasgos de personalidad u otros tipos de inteligencias clásicas). Sin duda, queda como reto conceptual articular estos enfoques comprehensivos que, además, contemplen la cultura y el contexto social de la persona como factores determinantes de lo que supone comportarse de manera emocionalmente inteligente.

En la primera década de nacimiento de la inteligencia emocional se realizaron esfuerzos considerables para tratar de medir de forma fiable las diferentes habilidades emocionales, ofreciendo enfoques evaluativos que aún hoy en día son utilizados y reconocidos de forma internacional. Otros equipos han sabido utilizar estos primeros enfoques de medición para avanzar en el desarrollo de herramientas que evalúen las habilidades no sólo con pruebas de papel y lápiz, sino con entornos audiovisuales. Posteriormente, se han liderado investigaciones en las dos décadas siguientes para demostrar la relativa contribución de las habilidades emocionales sobre diferentes aspectos importantes de la esfera humana, más allá de otros constructos psicológicos ya existentes. En la última década, el profesor Marc Brackett, que realizó la tesis doctoral con John Mayer y que, actualmente, trabaja con Peter Salovey y es director del Laboratorio de inteligencia emocional de la Universidad de Yale, ha seguido avanzando en este campo. Siguiendo la estela de sus dos mentores, ha desarrollado junto a su equipo un programa formativo internacional de desarrollo de inteligencia emocional de carácter transversal y basado en evidencias científicas (Modelo RULER), que actualmente está siendo implementado en numerosos países (Brackett et al., 2019).

A lo largo de este monográfico, a pesar de su limitación de espacio, queremos hacer un humilde homenaje a estos dos psicólogos americanos, creadores de una teoría vertebradora que ha permitido estructurar conocimientos y aunar hallazgos y áreas separadas de la cognición, la emoción, los estados afectivos, la neuropsicología o el funcionamiento interpersonal, entre otras. Los lectores encontrarán trabajos que han seguido la estela de Peter Salovey, Jack Mayer y su equipo, así como líneas de investigación más contemporáneas con aplicaciones a campos educativos, laborales y de la salud en la vida adulta. Somos conscientes de que estos trabajos no son una representación fidedigna de toda la amplia variedad de aplicaciones en un área tan fértil de investigación. Igualmente, anticipamos que no todas las cuestiones y áreas de aplicación y de intervención aún pendientes serán abordadas en este monográfico. No obstante, sí estamos seguros de que este monográfico llevará al lector a la reflexión, a nuevas preguntas sobre la importancia de ser emocionalmente inteligente en esta sociedad y a futuras aplicaciones en áreas de su interés. También esperamos que este monográfico lleve al lector a querer seguir ampliando la agenda investigadora en este campo, abriendo debates conceptuales, permitiendo contactar con los autores de estos trabajos, a la vanguardia en sus respectivas áreas, $y$, en definitiva, permitiendo nuevos espacios de comunicación y colaboración nacional e internacional. Creemos que estos trabajos contribuyen a resaltar la importancia aplicada del constructo de inteligencia emocional y plantean nuevos caminos y retos futuros que podrían ayudar a despertar el interés de nuevos investigadores y futuros trabajos académicos en universidades en forma de proyectos científicos o tesis doctorales que quieran seguir ahondando en esta fascinante área de la Psicología.

\section{Los contenidos de este monográfico homenaje a la inte- ligencia emocional}

A continuación, se presentan y se resumen las principales contribuciones de este monográfico homenaje al constructo de inteligencia emocional y que suponen un avance en diferentes áreas aplicadas de conocimiento. 


\section{Aportaciones sobre inteligencia emocional en el contex- to educativo: Estudios realizados en la etapa infanto- juvenil}

Desde un enfoque observacional de las aulas, investigadores de la Universidad Pedagógica y Tecnológica de Colombia analizan la importancia de la comunicación emocional no verbal en la infancia. Esta metodología examina los comportamientos gestuales de los alumnos y la coherencia con la comunicación verbal. Las investigaciones que evalúan la interacción y el comportamiento de los jóvenes en un ambiente escolar, proporcionan información sobre procesos dinámicos, percepción y expresión de emociones, motivaciones y lazos interpersonales que no podrían ser valorados con otros tipos de metodologías. Los hallazgos del trabajo de Porras-Cruz, Buitrago-Bonilla y GutiérrezTorres (2020) resaltan la necesidad de capacitar y desarrollar herramientas de identificación y análisis para los maestros con el fin de fomentar un buen clima del aula.

El estudio realizado por investigadores de la Universidad de Jaén (España) y del grupo Emo-é (España) examina las diferencias de género en cuanto al papel protector de la inteligencia emocional de los adolescentes y los efectos de este recurso personal sobre el burnout académico y la satisfacción vital. Considerando los hallazgos del trabajo de Berrios, Martos-Montes y Martos-Luque (2020), se muestra la necesidad de adoptar una perspectiva de género en la investigación en este campo. Estos resultados pueden contribuir a desarrollar programas de desarrollo emocional en el contexto educativo para mejorar el afrontamiento adaptativo del estrés e incrementar el bienestar en la adolescencia.

El estudio realizado por investigadores del grupo Applied Positive Lab de la Universidad de Málaga (España) aborda una temática de gran interés atendiendo al hecho de que los adolescentes pasan un mayor número de horas usando sus dispositivos tecnológicos. En el trabajo desarrollado por Arrivillaga, Rey y Extremera (2020) se examina el papel de la inteligencia emocional y otros factores de ajuste psicológico como predictores del uso problemático de smartphone. Los resultados de este trabajo apuntan a la necesidad de desarrollar intervenciones para el desarrollo de las competencias emocionales en la adolescencia para reducir el uso problemático de smartphone y las consecuencias negativas asociadas a este fenómeno. Asimismo, investigadores del mismo grupo de investigación de la Universidad de Málaga abordan en otro estudio las consecuencias negativas del ciberacoso en adolescentes y los recursos personales que pueden servir de protección. Debido a un mayor uso de los dispositivos tecnológicos y redes sociales, las conductas de ciberacoso han aumentado, conllevando graves secuelas para las víctimas. No obstante, estudios como el desarrollado por Chamizo-Nieto y Rey (2020) muestran cómo ciertos recursos personales de orden positivo como son las competencias emocionales y la gratitud, podrían servir para com- batir estas conductas disminuyendo los comportamientos de ciberagresión.

De forma paralela a los estudios anteriores sobre ciberacoso y uso problemático de los dispositivos tecnológicos, en otro estudio sobre acoso escolar tradicional, investigadores de la Universidad de Granada (España), del grupo de investigación Applied Positive Lab de la Universidad de Málaga y de la Universidad de Oporto (Portugal) examinan la importancia de emplear la inteligencia emocional en víctimas de acoso escolar para fomentar la satisfacción vital. Los hallazgos del trabajo de Quintana-Orts, Chamizo-Nieto, Rey y Neto (2020) muestran diferencias de género y la importancia de la inteligencia emocional como amortiguador de las consecuencias negativas de la victimización por acoso tradicional. En resumen, estos estudios destacan el papel de la inteligencia emocional en la adolescencia en relación con el uso de dispositivos tecnológicos y de posibles problemáticas asociadas a la convivencia escolar.

El problema de la agresividad en la adolescencia también puede ocurrir en el entorno familiar. De esta forma, investigadores del grupo OPIICS de la Universidad de Zaragoza (España) han evaluado la relación de la agresividad y la satisfacción con la familia en jóvenes, siendo la familia un agente socializador básico, y el papel de la inteligencia emocional como habilidad fundamental en relación con el autocontrol. Los resultados obtenidos en el trabajo de Larraz, Urbon, Antoñanzas y Salavera (2020) muestran que una base familiar sólida junto a la percepción subjetiva de satisfacción familiar favorece una gestión emocional saludable y un mayor número de comportamientos adaptativos. Esto se podría lograr gracias al fomento de la inteligencia emocional desde el propio núcleo familiar.

Además de los estudios empíricos descritos anteriormente, en este monográfico también se incluyen aportaciones teóricas y otras relacionadas con el diseño de programas para el desarrollo de la inteligencia emocional en la etapa infanto-juvenil.

El trabajo desarrollado por investigadores del Grupo en Investigación Psicopedagógica de la Universidad de Lleida (España) y de la Universidad de Barcelona (España), presenta un modelo teórico de resolución de conflictos a través de la educación emocional. En su trabajo, García Blanc, Gomis Cañellas, Ros Morente y Filella Guiu (2020) presentan un modelo por pasos que considera la toma de conciencia emocional, la reducción de intensidad emocional y la impulsividad, así como estrategias de regulación de las emociones, y un uso asertivo de la comunicación. Este modelo se ha gamificado a través de varios videojuegos para lograr un mejor acercamiento a los niños y adolescentes.

En cuanto a las propuestas de programas para el desarrollo de la inteligencia emocional en la infancia y la adolescencia, investigadores de la Universitat Jaume I (España) pre- 
sentan en su estudio una propuesta de desarrollo de habilidades emocionales a partir de los 3 años. En el estudio desarrollado por Murillo, Sánchez-Gómez y Bresó-Esteve (2020) se recoge el diseño de un programa dirigido al desarrollo de la inteligencia emocional dentro del núcleo familiar, con lo que se podría complementar el trabajo de educación emocional en las escuelas. En la misma línea, el trabajo llevado a cabo por investigadores del grupo EMINA de la Universitat de València (España) supone una contribución novedosa y de relevancia para el desarrollo socioemocional en la adolescencia mediante el uso de plataformas tecnológicas. En el estudio desarrollado por Barrera, Postigo-Zegarra, Tamarit, Gil-Gómez y Montoya-Castilla (2020) se aportan datos de interés acerca de la percepción de los participantes sobre la mejora de sus competencias socioemocionales y del atractivo de las actividades y el formato del Programa emoTIC. Los hallazgos de esta investigación apuntan a la necesidad de orientar la aplicación de los programas socioemocionales en el contexto educativo a las plataformas y los entornos virtuales para potenciar su eficacia.

\section{Aportaciones sobre inteligencia emocional en el contex- to educativo: Estudios realizados con el profesorado}

En cuanto a los trabajos sobre la inteligencia emocional de un agente clave del contexto educativo como es el profesorado, en este monográfico se han recogido propuestas empíricas y aplicadas dirigidas a la mejora de las competencias emocionales, las herramientas y el bienestar personal y laboral de los docentes.

En cuanto a los trabajos empíricos se han aportado dos trabajos sobre el bienestar del docente dentro y fuera del aula. Por un lado, el estudio de investigadores de la Universidad de Castilla-La Mancha (España) y la Universidad Española de Educación a Distancia (España) examina los efectos de la inteligencia emocional en relación con variables relevantes para un colectivo en riesgo psicosocial como los profesionales docentes: el afrontamiento resiliente y el bienestar subjetivo. Los hallazgos del trabajo de Rodríguez-Donaire, Luna, Pena y Cejudo (2020) apuntan a que la inteligencia emocional se asocia a un mayor uso de estrategias resilientes para el afrontamiento de estresores y a una mayor satisfacción vital. Así, estos resultados sugieren la incorporación de estos indicadores de recursos personales y bienestar en el desarrollo de programas de formación en educación emocional con docentes. Por otro lado, el estudio realizado por investigadores de la Universidad de Trás-os-Montes y Alto Douro (Portugal) y de la Universidade do Minho (Portugal) examina el papel de la inteligencia emocional del profesorado en relación con el uso de estrategias para abordar los conflictos en el aula. Los hallazgos del estudio de Valente y Lourenço (2020) han mostrado que las habilidades de inteligencia emocional del profesorado se asocian con el uso de estrategias más adecua- das para el manejo de conflictos. Estos resultados son de gran valor para el desarrollo de futuros programas de desarrollo de competencias emocionales con docentes para gestionar de forma más adecuada conflictos en el contexto educativo y promover relaciones positivas con el alumnado.

En cuanto al diseño y la aplicación de programas de intervención con el profesorado, el trabajo de investigadores del grupo EMINA de la Universitat de València aporta datos sobre el diseño y la implementación de un programa de educación emocional con profesionales de la enseñanza de distintos niveles de enseñanza reglada. En el trabajo desarrollado por Schoeps, Postigo-Zegarra, Mónaco, González y Montoya-Castilla (2020) se recogen datos acerca de la efectividad del programa para el desarrollo de competencias de inteligencia emocional y la mejora del bienestar que se han complementado con valoraciones acerca del impacto del programa sobre la mejora de habilidades emocionales y el desarrollo personal y profesional. Asimismo, el trabajo realizado por investigadores del grupo Applied Positive $L a b$ de la Universidad de Málaga aporta datos de interés sobre la implementación de un programa breve con profesorado novel. En el trabajo de Extremera, Mérida-López, Rey y Peláez-Fernández (2020) se muestra que los docentes noveles participantes incrementaron algunas habilidades emocionales claves, subrayando la utilidad y aplicabilidad percibida del programa para gestionar los conflictos en el aula. Aunque se requiere confirmación con diseños cuasiexperimentales y muestras más amplias, los resultados apoyan la idea de integrar módulos aplicados de formación emocional docente en los Másteres en Profesorado de Educación Secundaria como herramienta para mejorar su eficacia docente y lograr un mejor bienestar en el aula.

\section{Aportaciones sobre inteligencia emocional diversos ám- bitos: Estudios realizados en distintas esferas de la vida adulta}

Al dejar atrás la adolescencia, la etapa de la adultez implica enfrentarse a diversas situaciones estresantes como puede ser empezar la etapa de estudios universitarios o la etapa laboral. Estos nuevos cambios ponen a prueba las capacidades de gestión emocional de los individuos y su nuevo rol en la sociedad. En este monográfico se han presentado trabajos de revisión teórica y otros empíricos sobre el papel de la inteligencia emocional en diferentes áreas de la vida adulta.

El estudio realizado por investigadores del grupo Applied Positive Lab de la Universidad de Málaga y de la Universidad de Michigan (EEUU) examina el papel de diferentes recursos personales positivos, optimismo, gratitud e inteligencia emocional como predictores de indicadores positivos y negativos del bienestar universitario. En una muestra de 350 estudiantes universitarios españoles, los resultados 
mostraron que estos recursos son claves en una de las etapas de mayor estrés académico. El estudio de SánchezÁlvarez, Rey, Extremera y Chang (2020) refuerza la idea de que la inteligencia emocional aporta valor incremental sobre estos indicadores, más allá de la gratitud y el optimismo, sugiriendo la relativa importancia de la gestión emocional en esta etapa vital y apoyando la inclusión de módulos formativos universitarios sobre gestión emocional para afrontar los diversos estresores académicos a los que se enfrentan los jóvenes universitarios españoles.

Investigadores de la Universidad Autónoma de Nuevo León (México), de la Universitat Jaume I y de la Universidad Autónoma de Coahuila (México) han desarrollado una investigación en la que examinan cómo la inteligencia emocional y la espiritualidad puede influir en el apego al tratamiento y su influencia en problemas de adicciones al alcohol y drogas. En el estudio de Villarreal-Mata, Sánchez -Gómez, Navarro-Oliva, Bresó-Esteve y Pérez Rodríguez (2020) se resalta el carácter preventivo de las habilidades emocionales y la espiritualidad en adicciones. La inteligencia emocional ayudaría al control de impulsos mediante las estrategias de regulación emocional, lo que fomentaría los hábitos saludables. Además, las creencias espirituales ayudarían con el sentimiento de culpa que a su vez reduciría el consumo. Estos hallazgos pueden utilizarse para el desarrollo de estrategias y programas enfocados a mejorar el apego al tratamiento y prevenir recaídas.

El trabajo de revisión llevado a cabo por investigadores de grupos de investigación de la Universidad de La Laguna (Tenerife, España), la Universidad Complutense de Madrid (España) y la Universidad Pablo de Olavide de Sevilla (España) recoge diferentes conceptos de interés acerca del papel de las emociones en el sector hotelero. En el trabajo desarrollado por Barrientos-Báez, Caldevilla Domínguez, Martínez González y López Meneses (2020) se recogen evidencias acerca del papel de la inteligencia emocional como un recurso psicológico con beneficios para el departamento de RRHH debido a las implicaciones de estos profesionales en el desarrollo y la gestión de las personas implicadas en el sector hotelero. Entre las conclusiones del estudio se sugiere el desarrollo competencial de las habilidades emocionales en los planes de estudio previos al desempeño profesional en un sector profesional con altas demandas emocionales.

En el campo de la teoría del apego y el papel de la inteligencia emocional en personas adultas, investigadores de la Universidad del País Vasco (España) destacan la importancia de esta relación mediante la revisión de estudios científicos sobre el tema. Este estudio desarrollado por AlonsoArbiol y colaboradores (2020) permite revisar las aportaciones empíricas hasta la fecha y comprobar en varias muestras de población general, clínica, y personas empleadas cómo la inteligencia emocional se muestra asociada a la seguridad en el apego en estudios de distintas culturas, aportando un carácter universal a esta relación. Estos hallazgos proporcionan un acercamiento a la confluencia de los marcos teóricos del apego como de la inteligencia emocional y su desarrollo. Así lo muestran Rodríguez-Góngora y colaboradores (2020) en su estudio. Una investigación, realizada con familias en riesgo social, que trata de informar sobre el papel mediador de la inteligencia emocional en la formación de las estructuras familiares, donde la conjunción entre apego e inteligencia emocional es crucial para un desarrollo estructural adecuado, favorecedor de un clima familiar positivo.

Finalmente, el trabajo de revisión del equipo de la Universidad de Jaén aporta una ilustración de resultados acerca de los efectos de la inteligencia emocional en contextos psicosociales aplicados. Este estudio de Berrios, Pulido-Martos, Augusto-Landa y Lopez-Zafra (2020) describe e integra aportaciones de su grupo de investigación para aportar una panorámica acerca del estado actual de la cuestión sobre el papel de las habilidades de inteligencia emocional en el ajuste en la adolescencia, el entorno docente o sanitario o la intervención psicosocial. Mediante la revisión pormenorizada por ámbitos aplicados, en este trabajo se revisan algunas limitaciones del estado actual de la investigación en inteligencia emocional y se proponen algunas líneas de trabajo futuras que merecen especial atención.

\section{Aportaciones de este monográfico para el presente y el futuro de la inteligencia emocional}

Los trabajos incluidos en este monográfico abordan temáticas de gran relevancia e interés que ilustran el desarrollo del constructo de inteligencia emocional a lo largo de tres décadas de investigación. A continuación se presentan algunas futuras líneas de trabajo básico y aplicado en cuanto a las posibles direcciones que podrían abordar los equipos de investigación nacionales e internacionales que trabajen sobre el constructo de inteligencia emocional.

El desarrollo de las etapas de la infancia y la adolescencia se consideran cruciales debido a que se encuentran llenas de múltiples transiciones estresantes como cambios físicos, educativos, sociales y sexuales. Estos cambios favorecen la experimentación de factores estresantes negativos que amenazan la salud emocional, precisamente en etapas del desarrollo en los cuales aún se produce un proceso de maduración de las habilidades emocionales. Unas apropiadas habilidades para gestionar las emociones permitirán a los niños y adolescentes hacer frente a las dificultades sobrevenidas por los cambios, fomentando el bienestar y aliviando el desajuste psicológico asociado al estrés. Así pues, estas etapas son clave para un fomento de la inteligencia emocional tanto desde los centros educativos como de los propios núcleos familiares. Tal y como se desprende en este monográfico, padres y educadores han de contar entre sus herramientas para la educación con programas fiables y bien 
fundamentados para el correcto desarrollo de las capacidades emocionales de niños y adolescentes. Por ello, futuras líneas de trabajo podrían ir dirigidas hacia la consolidación de programas de intervención en la etapa infanto-juvenil con el objetivo de desarrollar unas capacidades emocionales óptimas. Para alcanzar una mayor comprensión de la inteligencia emocional a estas edades se hace necesario adaptar y crear nuevas formas de medición de las capacidades emocionales, recurriendo a instrumentos y metodologías novedosos y atractivos que conecten con los jóvenes (p.ej., gamificación). Las futuras líneas de trabajo a estas edades deberían contar con una proyección dentro del ámbito educativo, ya que muchos de los déficits de ajuste psicosocial afectan a indicadores académicos o presentes en los centros educativos. Considerando los déficits de inteligencia emocional asociados a problemas relacionados con la agresividad, relaciones sociales entre iguales y dificultades para superar los eventos estresantes, los centros educativos son llamados a formar parte activa en la educación emocional de los niños y adolescentes, siendo necesario la implicación de todos los agentes educativos en el comprehensivo desarrollo de las habilidades emocionales a estas edades. Concretamente, se destaca la importancia de los docentes y padres como modelos de gestión emocional, cuyo papel también debiera ir dirigido hacia el fomento de aulas y hogares emocionalmente inteligentes.

En relación con los trabajos realizados con profesionales de la educación, las futuras investigaciones con este colectivo en riesgo psicosocial deberían atender a las demandas profesionales relacionadas con nuevas realidades de trabajo como la enseñanza bimodal. Asimismo, resulta necesario dar respuesta a las necesidades competenciales de los docentes para acompañar y proporcionar apoyo al alumnado tras situaciones de crisis vitales como la pandemia global vivida desde principios de 2020. Considerando el papel del docente como modelo de gestión emocional para el alumnado, es necesario que los futuros estudios en esta área examinen los efectos de contagio entre los eventos personales y profesionales y el estrés y desgaste profesional asociado. De esta forma, se podría aportar evidencia contrastada sobre el efecto de la gestión emocional de los profesionales docentes sobre el clima del aula y el bienestar del alumnado en situaciones de estrés.

Los futuros trabajos sobre inteligencia emocional del profesorado deberían focalizar la atención en el papel longitudinal de las competencias emocionales y el análisis pormenorizado de procesos intrapersonales e interpersonales de forma que se permita un conocimiento más detallado de su utilidad para reducir el estrés laboral. Esto permitiría conocer en mayor medida qué estrategias usan los docentes emocionalmente inteligentes para promover y mantener el bienestar docente según determinadas situaciones demandantes. Por ejemplo, examinar el papel de la inteligencia emocional en relación con variables macro relacionadas con la conflictividad del centro educativo y las relaciones interpersonales con estudiantes y familias. Además, las futuras investigaciones en este campo deberían abordar el uso de medidas que superen las limitaciones de los autoinformes. Por ejemplo, convendría incorporar el uso de medidas en tiempo real e indicadores psicofisiológicos para profundizar en los efectos de la adecuada gestión emocional en el aula y en el contexto educativo. Finalmente, los profesionales de la enseñanza deberían contar con programas de intervención rigurosos y basados en la evidencia que les permitan incorporar herramientas y estrategias para gestionar adecuadamente las nuevas problemáticas y realidades asociadas al contexto docente. Estos programas deberían incidir en la relación profesor-estudiante y abordar las agresiones al profesorado desde un enfoque preventivo y de fomento de la conectividad con el alumnado.

En la vida adulta, las futuras investigaciones en inteligencia emocional deberán servir para proporcionar recursos de afrontamiento ante las principales situaciones de estrés que surgen en esta etapa (p.ej., enfermedades crónicas, desempleo, divorcio, etc.). Igualmente, queda por analizar si los adolescentes que han recibido formación en inteligencia emocional son, posteriormente, adultos con un mayor ajuste psicosocial y con indicadores más positivos de salud, empleabilidad y de relaciones interpersonales. De hecho, una cuestión relacionada sería valorar el impacto económico a largo plazo para la vida adulta que supondría fomentar la inteligencia emocional. En EEUU se ha encontrado que cada inversión de un dólar supone un beneficio de 11 dólares para la sociedad, reduciendo los niveles de criminalidad juvenil y disminuyendo los costes sanitarios y sociales (Belfield et al., 2015). En Bélgica se ha encontrado que cada inversión formativa dedicada a mejorar un $1 \%$ la inteligencia emocional corresponde a un descenso de un $1 \%$ en gastos sanitarios. El retorno de la inversión varía en función del nivel educativo de las personas, siendo el retorno más elevado en aquellos con niveles educativos más bajos (Mikolajczak y Van Bellegem, 2017). Desafortunadamente, aún no contamos con datos sobre esta cuestión ni en España ni en países latinoamericanos. Por ello, sería de gran interés para las políticas de salud pública esclarecer el impacto a medio y largo plazo de mejorar la inteligencia emocional y especialmente, indagar las circunstancias sociales en las cuales esta inversión podría redundar en mayores beneficios en la vida adulta.

Considerando que la etapa adulta es la antesala de la tercera edad, y dado el incremento de nuestra esperanza de vida, se deben desarrollar nuevas líneas de trabajo focalizadas en examinar si las personas adultas con un nivel adecuado de inteligencia emocional muestran correlatos más positivos de salud cuando llegan a la tercera edad, incluso a pesar de los eventos vitales estresantes y los cambios físicos, fami- 
liares y sociales asociados a esta etapa. Quizás la investigación nos muestre que el desarrollo de las habilidades emocionales durante la edad adolescente y la vida adulta podrían reducir la dependencia e, incluso, los costes sociales y sanitarios cuando las personas llegan a la vejez. Es necesario resaltar que el COVID-19 ha generado la mayor crisis de salud en lo que llevamos de siglo XXI, aunque para los investigadores en el campo de la inteligencia emocional se abre un marco incomparable para analizar las habilidades emocionales y los procesos de afrontamiento efectivos que estén llevando a cabo las personas ante el estrés por la pandemia y su repercusión sobre indicadores de salud física y bienestar psicosocial.

Si bien la inteligencia emocional ha recorrido un largo camino desde su primer artículo científico en 1990, los próximos 30 años estarán llenos de retos y desafíos en el estudio de las emociones y la inteligencia que matizarán las investigaciones realizadas sobre la utilidad en el campo educativo, clínico y organizacional, aportando nuevas evidencias transculturales, novedosas herramientas de medición, aprovechando los avances tecnológicos, y programas aplicados innovadores para colectivos con necesidades afectivas aún sin explorar. Esperamos que este monográfico sirva para poner un granito de arena en esa dirección. Si, gracias a él, algunas futuras promesas investigadoras de cualquier parte del mundo se animan a continuar el legado iniciado por Peter Salovey y John Mayer, habremos conseguido con creces el objetivo de este volumen especial de la revista Know and Share Psychology.

Por último, queremos expresar nuestro más sincero agradecimiento como editores invitados de este homenaje a los padres del constructo, Peter Salovey y John Mayer, por su constante y rigurosa labor de desarrollo científico en esta fascinante área de la Psicología y por aportar su tiempo y dedicación con unas emotivas palabras en la parte inicial de este monográfico. Muchas gracias de corazón por todo. Igualmente, agradecemos a todos los expertos nacionales $\mathrm{e}$ internacionales, colegas y amigos de investigación, su tiempo y esfuerzo por aceptar la invitación de compartir sus líneas actuales de investigación en inteligencia emocional generadas en sus laboratorios. También queremos mostrar nuestra gratitud a los revisores anónimos por ser tan constructivos y rigurosos, así como agradecerles su esfuerzo desinteresado y su dedicación que han sido claves en este monográfico. Por último, como no podía ser de otra manera, nuestro reconocimiento y agradecimiento más sincero a la invitación de Juan Carlos Rodríguez y Josefina Rodríguez, como editores de la revista Know and Share Psychology, por animarnos y creer en que se podría realizar este homenaje antes de que acabase $2020 \mathrm{y}$, de esta forma, poder realizar este tributo al trigésimo aniversario del constructo de inteligencia emocional. Sin su estupenda y eficiente labor editorial y su apoyo incondicional no hubiera sido posible acabar a tiempo este sentido homenaje.

\section{Referencias}

Alonso-Arbiol, I., Bretaña, I., Mateos-Pérez, E., Páez, D., Torres-Gómez, B., Pascual, A., Conejero, S. y Gallarin, M. (2020). Inteligencia emocional y apego en población adulta: Una revisión sistemática. Know and Share Psychology, 1(4), 77-90.

Arrivillaga, C., Rey, L. y Extremera, N. (2020). Uso problemático del smartphone y ajuste psicológico en adolescentes: El papel clave de la inteligencia emocional. Know and Share Psychology, 1(4), 147-158.

Barrera, U., Postigo-Zegarra, S., Tamarit, A., Gil-Gómez, J.A. y Montoya-Castilla, I. Desarrollo de competencias socio-emocionales mediante plataforma tecnológica (Programa emoTIC): Valoración de los beneficios del programa según los participantes. Know and Share Psychology, 1(4), 241-255.

Barrientos-Báez, A., Caldevilla Domínguez, D., Martínez González, J.-A. y López Meneses, E. (2020). Gestión de las emociones: Departamento de RRHH en hotelería. Know and Share Psychology, 1(4), 135146.

Belfield, C., Bowden B., Klapp, A., Levin, H., Shand, R. y Zander, S. (2015). The economic value of social and emotional learning. Journal of Benefit-Cost Analysis, 6(3), 508-544. https://doi.org/10.1017/ bca. 2015.55

Berrios, M.P., Martos-Montes, R. y Martos-Luque, R. (2020). Influencia del género en las relaciones entre la inteligencia emocional, el estrés académico y la satisfacción de los estudiantes. Know and Share Psychology, 1(4), 229-240.

Berrios, M.P., Pulido-Martos, M., Augusto-Landa, J.M. y Lopez-Zafra, E. (2020). Inteligencia emocional en distintos colectivos: Aportaciones del grupo TEAM $^{+}$. Know and Share Psychology, 1(4), 19-37

Brackett, M.A., Bailey, C.S., Hoffmann, J.D. y Simmons, D.N. (2019). RULER: A theory-driven, systemic approach to social, emotional, and academic learning. Educational Psychologist, 54(3), 144-161. https://doi.org/10.1080/00461520.2019.1614447

Chamizo-Nieto, M.T. y Rey, L. (2020). Ciberacoso y recursos personales en adolescentes: Las competencias emocionales y la gratitud como factores de protección ante las conductas de ciberagresión. Know and Share Psychology, 1(4), 113-122.

Extremera, N., Mérida-López, S., Rey, L. y PeláezFernández, M.A. (2020). Programa "CRECIENDO" (Creando Competencias de Inteligencia Emocional en Nuevos Docentes): Evidencias 
preliminares y su utilidad percibida en la formación inicial del profesorado de Secundaria. Know and Share Psychology, 1(4), 201-210.

Garcia Blanc, N., Gomis Cañellas, R., Ros Morente, A. y Filella Guiu, G. (2020). El proceso de gestión emocional: Investigación, aplicación y evaluación. Know and Share Psychology, 1(4), 39-49.

Gardner, H. (1983). Frames of Mind: The Theory of Multiple Intelligences. Basic Books.

Goleman, D. (1995). Emotional Intelligence: Why it Can Matter More Than IQ. Bloomsbury.

Larraz, N. Urbon, E., Antoñanzas, J.L. y Salavera, C. (2020). La satisfacción con la familia y su relación con la agresividad y la inteligencia emocional en adolescentes. Know and Share Psychology, 1(4), 171-179.

Leuner, B. (1966). Emotionale intelligenz und emanzipation (Emotional intelligence and emancipation). Praxis der Kinderpsychologie und Kinderpsychiatry, 15, 196-203.

Mayer, J.D., Caruso, D.R. y Salovey, P. (2016). The ability model of emotional intelligence: Principles and updates. Emotion Review, 8(4), 290-300. https:// doi.org/10.1177/1754073916639667

Mikolajczak, M. y Van Bellegem, S. (2017). Increasing emotional intelligence to decrease healthcare expenditures: How profitable would it be? Personality and Individual Differences, 116, 343-347. https:// doi.org/10.1016/j.paid.2017.05.014

Murillo, A.L., Sánchez-Gómez, M. y Bresó-Esteve, E. (2020). Inteligencia emocional en familia: Un programa formativo para padres e hijos de 3 a 6 años. Know and Share Psychology, 1(4), 61-75.

Porras-Cruz, L.T., Buitrago-Bonilla, R.E. y GutiérrezTorres, A.M. (2020). Comunicación emocional no verbal en la infancia, análisis en el aula desde la metodología observacional. Know and Share Psychology, 1(4), 211-228.

Quintana-Orts, C., Chamizo-Nieto, M.T., Rey, L. y Neto, F. (2020). Bienestar en víctimas de acoso: ¿puede la inteligencia emocional marcar una diferencia en función del género de los adolescentes? Know and Share Psychology, 1(4), 189-200.

Rodríguez-Góngora, J., Rodríguez-Rodríguez, J.C. y Rodríguez-Rodríguez, J.-A. La inteligencia emocional como variable mediadora en la formación de estructuras familiares equilibradas. Know and Share Psychology, 1(4), 51-59.
Rodríguez-Donaire, A., Luna, P., Pena, M. y Cejudo, J. (2020). El papel de la inteligencia emocional en el afrontamiento resiliente y la satisfacción con la vida en docentes. Know and Share Psychology, 1(4), 181 -188 .

Salovey, P. y Mayer, J.D. (1990). Emotional intelligence. Imagination, Cognition, and Personality, 9, 185211. https://doi.org/10.2190\%2FDUGG-P24E$52 \mathrm{WK}-6 \mathrm{CDG}$

Sánchez-Álvarez, N., Rey, L., Extremera, N. y Chang, O.D. (2020). Más allá del papel de los recursos personales positivos en el bienestar de estudiantes universitarios españoles: ¿Importa la inteligencia emocional? Know and Share Psychology, 1(4), 91-101.

Schoeps, K., Postigo-Zegarra, S., Mónaco, E., González, R. y Montoya-Castilla, I. (2020). Valoración del Programa de Educación Emocional para Docentes (MADEMO). Know and Share Psychology, 1(4), 159-170.

Thorndike, E.L. (1920). Intelligence and its uses. Harper's Magazine, 140, 227-235.

Valente, S. y Lourenço, A.A. (2020). La inteligencia emocional marca la diferencia: el impacto de las habilidades de inteligencia emocional del profesorado en las estrategias de manejo de conflictos en el aula. Know and Share Psychology, 1(4), 123-134.

Villarreal-Mata, J.L., Sánchez-Gómez, M., Navarro-Oliva, E.I.P., Bresó-Esteve, E. y Pérez Rodríguez, E. (2020). Inteligencia emocional y espiritualidad en el apego al tratamiento de adultos con adicciones al alcohol y drogas. Know and Share Psychology, 1 (4), 103-111. 